\title{
Expression of $\boldsymbol{N}$-Acetylgalactosamine 4-Sulfate 6-O-Sulfotransferase Involved in Chondroitin Sulfate Synthesis Is Responsible for Pulmonary Metastasis
}

\author{
Shuji Mizumoto, ${ }^{1}$ Moto Watanabe, ${ }^{1}$ Shuhei Yamada, ${ }^{1,2}$ and Kazuyuki Sugahara ${ }^{1}$ \\ ${ }^{1}$ Laboratory of Proteoglycan Signaling and Therapeutics, Frontier Research Center for Post-Genomic Science and Technology, \\ Graduate School of Life Science Hokkaido University, West-11, North-21, Kita-ku, Sapporo, Hokkaido 001-0021, Japan \\ ${ }^{2}$ Department of Pathobiochemistry, Faculty of Pharmacy, Meijo University, 150 Yagotoyama, Tempaku-ku, Nagoya 468-8503, Japan
}

Correspondence should be addressed to Kazuyuki Sugahara; k-sugar@sci.hokudai.ac.jp

Received 27 November 2012; Accepted 20 December 2012

Academic Editor: Davide Vigetti

Copyright (C) 2013 Shuji Mizumoto et al. This is an open access article distributed under the Creative Commons Attribution License, which permits unrestricted use, distribution, and reproduction in any medium, provided the original work is properly cited.

\begin{abstract}
Chondroitin sulfate (CS) containing E-disaccharide units, glucuronic acid- $\mathrm{N}$-acetylgalactosamine $(4,6$ - $O$-disulfate), at surfaces of tumor cells plays a key role in tumor metastasis. However, the molecular mechanism of the metastasis involving the CS chaincontaining E-units is not fully understood. In this study, to clarify the role of E-units in the metastasis and to search for potential molecular targets for anticancer drugs, the isolation and characterization of Lewis lung carcinoma (LLC) cells stably downregulated by the knockdown for the gene encoding $\mathrm{N}$-acetylgalactosamine 4 - $\mathrm{O}$-sulfate 6-O-sulfotransferase (GalNAc4S-6ST), which is responsible for the formation of E-units in CS chains, were performed. Knockdown of GalNAc4S-6ST in LLC cells resulted in a reduction in the proportion of E-units, in adhesiveness to extracellular matrix adhesion molecules and in proliferation in vitro. Furthermore, the stable downregulation of GalNAc4S-6ST expression in LLC cells markedly inhibited the colonization of the lungs by inoculated LLC cells and invasive capacity of LLC cells. These results provide clear evidence that CS chain-containing E-units and/or GalNAc4S-6ST play a crucial role in pulmonary metastasis at least through the increased adhesion and the invasive capacity of LLC cells and also provides insights into future drug targets for anticancer treatment.
\end{abstract}

\section{Introduction}

Chondroitin sulfate (CS) is a sulfated glycosaminoglycan (GAG) that covalently attaches to core proteins to form CSproteoglycans (CS-PGs) [1, 2]. CS/DS-PGs are ubiquitous in extracellular matrices (ECMs) and at cell surfaces in various tissues and regulate various physiological events such as cell proliferation, cytokinesis, morphogenesis, and viral infections through interaction with various proteins $[3,4]$. Furthermore, CS-PGs at the tumor cell surface and in the ECM are related to metastatic potential and facilitate tumor invasion by enhancing integrin-mediated cell adhesion, motility, and intracellular signaling [5-8]. Interestingly, the binding of P-selectin to a tumor cell surface depends on the expression of the CHST11 gene encoding chondroitin 4-O-sulfotransferae-1 (C4ST-1), and CSPG4 (also known as melanoma-associated CSPG) serves as a P-selectin ligand through its CS side chains and participates in the binding of P-selectin to highly metastatic breast cancer cells [9]. Further, the expression of a PG, versican, is upregulated in various types of tumors including lung cancer, as a macrophage activator that acts through Toll-like receptor- 2 and its co-receptors Toll-like receptor- 6 and CD14 [10].

The sugar backbone of CS chains is a linear polysaccharide consisting of repeating disaccharide units, [-4GlcUA $\beta 1$ 3 GalNAc $\beta 1-]_{n}$, where GlcUA and GalNAc represent Dglucuronic acid and $\mathrm{N}$-acetyl-D-galactosamine, respectively, [4]. CS chains are modified by specific sulfotransferases, which catalyze the transfer of a sulfate group from $3^{\prime}$ phosphoadenosine $5^{\prime}$-phosphosulfate to C-2 of GlcUA and C-4 and/or C-6 of GalNAc. The combination of sulfated positions results in several disaccharide units with different 
sulfation patterns and yields enormous structural diversity in terms of sequence $[3,11]$. For instance, monosulfated GlcUA-GalNAc(4-O-sulfate) and GlcUA-GalNAc(6-Osulfate) disaccharides, abbreviated as A- and C-units, are involved in the differentiation of chondrocytes and the development of a spine, respectively [12-15]. Representative disulfated disaccharide units are $\operatorname{GlcUA}(2-O$-sulfate)GalNAc(6-O-sulfate) (D-units) and GlcUA-GalNAc(4-O-, 6$O$-disulfate) (E-units), which function in neuritogenesis [16$18]$ and viral infections $[19,20]$, respectively.

Recently, it has been reported that the expression of Eunit-containing structures recognized by an anti-CS-E phage display antibody, GD3G7 [21,22], is increased in ovarian and pancreatic cancer tissues, resulting in alterations in tumor growth and tumor cell motility through the regulation of the signaling of vascular endothelial growth factor (VEGF) and the cleavage of CD44, respectively [22, 23]. Further, the expression of GalNAc 4-O-sulfate 6-O-sulfotransferase (GalNAc4S-6ST), which transfers a sulfate group to position 6 of GalNAc(4-O-sulfate) in A-disaccharide units formed by C4ST and is responsible for the formation of E-units [24, $25]$, is increased in colorectal cancer tissues compared with paired normal mucosa [26]. Thus, these observations appear to suggest that E-units in CS chains are upregulated in tumor tissues compared to normal tissues.

Moreover, we demonstrated that the expression of GalNAc4S-6ST and the proportion of disulfated E-disaccharides are increased in highly metastatic compared to low metastatic Lewis lung carcinoma (LLC) cells [27]. The colonization by intravenously injected LLC cells of mouse lungs was efficiently inhibited by preinjected CS-E polysaccharides, rich in E-units, derived from squid cartilage and by the anti-CS-E phage display antibody, GD3G7 [27], suggesting GalNAc4S-6ST and/or E-unit-containing CS chains to be involved in the pulmonary metastasis of LLC cells. In addition, the GAG-binding receptor in mouse lung was recently identified as Receptor for Advanced Glycation Endproducts (RAGE), which showed high affinity toward CS-E and heparan sulfate chains [28]. However, the exact structural features of GAGs remain to be investigated especially because RAGE could interact with both CS-E and heparan sulfate with high affinity [28]. In the present study, to clarify the role of E-units in metastasis, the isolation and characterization of LLC cells stably downregulated for the gene encoding GalNAc4S-6ST by knockdown using short hairpin RNA were performed.

\section{Materials and Methods}

2.1. Materials. The following sugars and enzymes were purchased from Seikagaku Biobusiness Corp. (Tokyo, Japan): CS-A from whale cartilages; CS-E from squid cartilages; six unsaturated standard disaccharides derived from CS; chondroitinase ABC (EC 4.2.2.20) from Proteus vulgaris; chondroitinase AC-II (EC 4.2.2.5) from Arthrobacter aurescens. Short hairpin RNA- (shRNA-) expressing plasmids (cat no.: sc-145317-SH) specific to mouse GalNAc4S6ST, which target 5'-CUACAAUGUGGGAUAACAA-3',
$5^{\prime}$-CAAGACACCCUUAGAAUGU-3', and 5'-GAACACUCGUGCUUAUACU-3' and scrambled nucleotide sequence-containing control-shRNA plasmids (cat no.: sc108060), shRNA transfection reagent, and shRNA plasmid transfection medium were purchased from Santa Cruz Biotechnologies, Inc (Santa Cruz, CA, USA).

2.2. Animals and Cell Lines. Seven-week-old male C57BL/6J mice and LLC cells were obtained from Japan SLC (Hamamatsu, Japan) and RIKEN Cell Bank (Tsukuba, Japan), respectively. All the experiments were performed under the experimental protocol approved by the local animal care committee of Hokkaido University.

2.3. Isolation of LLC Clones Stably Downregulated for the Expression of GalNAc4S-6ST. The GalNAc4S-6ST (Chst15) and control shRNA plasmids were individually transfected according to the manufacturer's instructions. The resultant puromycin-resistant colonies were subcultured on a 96-well culture plate by limiting dilution at a low density ( 1 cell/well), and were propagated.

2.4. Quantitative Real-Time PCR. Total RNA was extracted from each clone using an RNA isolation kit, illustra RNAspin Midi (GE Healthcare, Buckinghamshire, UK). Each cDNA was synthesized from $\sim 1 \mu \mathrm{g}$ of the total RNA using Moloney murine leukemia virus-reverse transcriptase (Promega, Madison, WI, USA) and an oligo $(\mathrm{dT})_{16}$ primer (Hokkaido System Science, Sapporo, Japan). The primer sequences used were as follows: for GalNAc4S-6ST (153bp), 5'TATGACAACAGCACAGACGG-3' (forward) and $5{ }^{\prime}$-TGCAGATTTATTGGAACTTGCGAA-3' (reverse); for glyceraldehyde-3-phosphate dehydrogenase (G3pdh) (205bp), 5'CATCTGAGGGCCCACTG-3' and 5'-GAGGCCATGTAGGCCATGA-3. Quantitative real-time PCR was performed using a Brilliant II SYBER Green QPCR master mix in Mx3005P real-time QPCR (Agilent Technologies, Santa Clara, CA, USA). The level of GalNAc4S-6ST mRNA was normalized to that of the transcript of G3pdh.

\subsection{Analysis of Disaccharide Composition of CS/DS Chains Isolated from LLC Cells. To obtain evidence that the knock- down of GalNAc4S-6ST results in a reduction in E-units [GlcUA-GalNAc(4-O-, 6-O-disulfates)] in CS chains from LLC cells, the CS disaccharide composition of each clone was determined as described previously [29, 30].}

2.6. Assays for Lung Metastasis. To investigate the effects of the knockdown of GalNAc4S-6ST on experimental tumor metastasis, the control shRNA- and GalNAc4S-6STshRNA/LLC cells $\left(1 \times 10^{6}\right.$ cells/mouse $)$ were injected into a lateral tail vein of $\mathrm{C} 57 \mathrm{BL} / 6$ mice as described in [27]. Three weeks after the injection, the animals were sacrificed, and the number of visible and parietal nodules in the lung was counted by two observers in a blinded fashion. 
2.7. Cell Adhesion Assay. Plastic cover slips $(10 \times 10 \mathrm{~mm})$ were precoated with $10 \mu \mathrm{g} / \mathrm{mL}$ of laminin (Invitrogen), fibronectin, or type IV collagen (BD Biosciences, San Jose, CA, USA) overnight at $4^{\circ} \mathrm{C}$ and then washed with phosphatebuffered saline twice. The control shRNA and GalNAc4S6ST-shRNA/LLC cells were seeded on cover slips in 24-well plates at $5 \times 10^{4}$ cells $/ \mathrm{mL}$ in serum-free Dulbecco's modified Eagle's medium (DMEM) and incubated for $1 \sim 2 \mathrm{~h}$ at $37^{\circ} \mathrm{C}$. The supernatant with nonadherent cells was removed by three washes with a warmed culture medium. Attached cells were fixed, stained with the Diff-Quik staining kit (Sysmex International Reagents Co., Kobe, Japan), and counted in an area of $2 \mathrm{~mm}^{2}$.

2.8. Cell Migration and Invasion Assays. The ability of GalNAc4S-6ST-shRNA/LLC cells to invade and migrate was assessed using the $\mathrm{BD}$ BioCoat chamber with or without Matrigel (BD Biosciences) in vitro, respectively. The lower chambers were filled with DMEM containing 10\% FBS, and single cell suspensions of LLC cells $\left(2 \times 10^{4}\right.$ cells/500 $\left.\mu \mathrm{L}\right)$ in serum-free DMEM were placed in the upper chamber. After incubation for $26 \mathrm{~h}$, the cells, which migrated or invaded through the membrane alone or the Matrigel-coated membrane, respectively, and remained bound to the underside of the membranes, were stained with the Diff-Quik and counted in five random microscopic fields/filters.

2.9. Cell Proliferation Assay. The control shRNA and GalNAc4S-6ST-shRNA/LLC cells were seeded in 96-well plates at 2,000 cells/well in DMEM containing 10\% FBS and cultured for various periods. The number of living cells was measured at each time point using TetraColor One (Seikagaku Biobusiness Co.) according to the manufacturer's instructions. Triplicate cultures were used for each sample. After incubation for $1 \mathrm{~h}$ at $37^{\circ} \mathrm{C}$, the developed color was measured at $450 \mathrm{~nm}$ using a microplate reader (Bio-Rad, Hercules, CA, USA).

\section{Results}

3.1. Isolation and Characterization of the LLC Cells Expressing shRNAs Specific for GalNAc4S-6ST. To isolate the clones of LLC cells in which the GalNAc4S-6ST gene was suppressed, the vectors expressing shRNAs, which also contain the puromycin-resistance gene, specific for mouse GalNAc4ST6ST were introduced into the cells. Fifty-seven LLC clones resistant to puromycin were isolated (LLC-4S6ST-shRNA). To examine the efficacy of the knockdown of GalNAc4S6ST by specific shRNA, quantitative real-time PCR was conducted after the extraction of total RNA from ten randomly selected clones, followed by the synthesis of the cDNA. The isolated LLC-4S6ST-shRNA clones (nos. 7, 17, and 23) showed the downregulation of GalNAc4S-6ST (30 40\% of the control-shRNA clones) (Figure 1(a)). Thus, these three clones were utilized for further analyses.

To further characterize the effects of the knockdown of the GalNAc4S-6ST gene on the amount of E-units, the disaccharide composition of CS chains, which were prepared from each clone as a GAG-peptide fraction, was determined. Representative chromatograms are shown in Figures 1(b) and $1(\mathrm{c})$, and the composition and amounts of the disaccharides are summarized in Table 1. The data obtained from the digest of the GAG-peptides using a mixture of chondroitinases $\mathrm{ABC}$ and $\mathrm{AC}-\mathrm{II}$ revealed that the low sulfated disaccharide, HexUA-GalNAc(4-O-sulfate) (A), where HexUA represents hexuronic acid ( $\beta$-GlcUA or $\alpha$-iduronic acid), was a major disaccharide unit, $\sim 94 \%$ (Table 1 ), and HexUA-GalNAc(4-, 6-O-disulfate) (E) accounted for $\sim 6 \%$ of all the disaccharides in the LLC-control-shRNA cells consistent with a previous report [28]. On the other hand, a drastic reduction in the proportion of E-units, to $0.5 \sim 1.9 \%$, was observed in the LLC-4S6ST-shRNA clones (nos. 7, 17, and 23) compared to the LLC-control-shRNA cells (Table 1). Thus, these clones were utilized to further experiments. It should be noted that the amounts of total disaccharides recovered were less in GalNAc4S-6ST-shRNA clones (213, 323 , and $246 \mathrm{pmol} / \mathrm{mg}$ acetone powder) compared to wildtype cells and control-shRNA clones (754, 1348, 924, and $534 \mathrm{pmol} / \mathrm{mg}$ acetone powder), suggesting that GalNAc4S6ST or CS chains containing E-units may affect the amounts or lengths of CS chains, or other CS-biosynthetic enzymes. In contrast, bone marrow-derived mast cells from the knockout mice of GalNAc4S-6ST synthesized larger CS chains than the wild type, and levels of the chondroitin 4-O-sulfotransferase1 and chondroitin 6-O-sulfotransferase- 1 transcripts in the homozygous mutant mice were higher than those in the wild type [25]. Thus, the contrasting effect on the biosynthesis of CS by the expression of GalNAc4S-6ST in LLC cells remains to be elucidated.

\subsection{Effects of the Knockdown of the GalNAc4S-6ST Gene in} LLC Cells on Pulmonary Metastasis. To assess the influence of the knockdown of the GalNAc4S-6ST gene and the resulting reduction of E-units in LLC cells on pulmonary metastasis, the LLC-4S6ST-shRNA clones were individually inoculated into mice via a tail vein. Three weeks later, the mice were sacrificed, and pulmonary metastasis was evaluated by counting tumor foci on the lung surface and weighing the lung tissues. As expected, the knockdown of GalNAc4S-6ST drastically reduced the metastasis of LLC cells compared with that in mice injected with the LLC-control-shRNA (Figure 2), suggesting a crucial role for the cell surface CS chains containing E-units in the pulmonary metastasis of LLC cells.

3.3. Characterization of the LLC-4S6ST-shRNA Cells In Vitro. LLC cells are frequently utilized as a model for experimental lung metastasis [31]. After their inoculation into the tail of mice, LLC cells reach the lung and may bind to the surface of the vascular endothelium through adhesion to ECM molecules [32-34]. Hence, to assess the change in the adhesiveness of LLC cells by the knockdown of GalNAc4S-6ST, the adhesive capacity of the LLC-4S6STshRNA was examined using adhesion molecules in ECM including laminin, fibronectin, and type IV collagen. The number of LLC-4S6ST-shRNA cells adhering to laminin or fibronectin but not to type IV collagen was significantly 


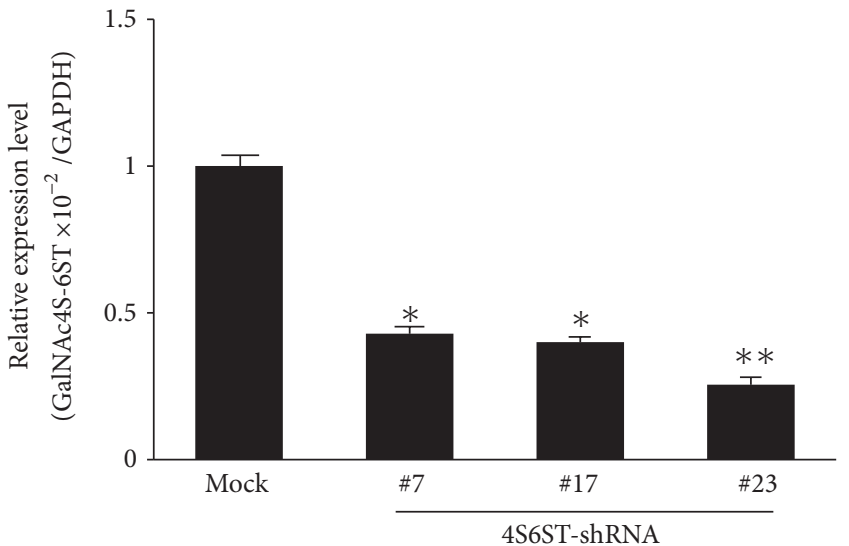

(a)

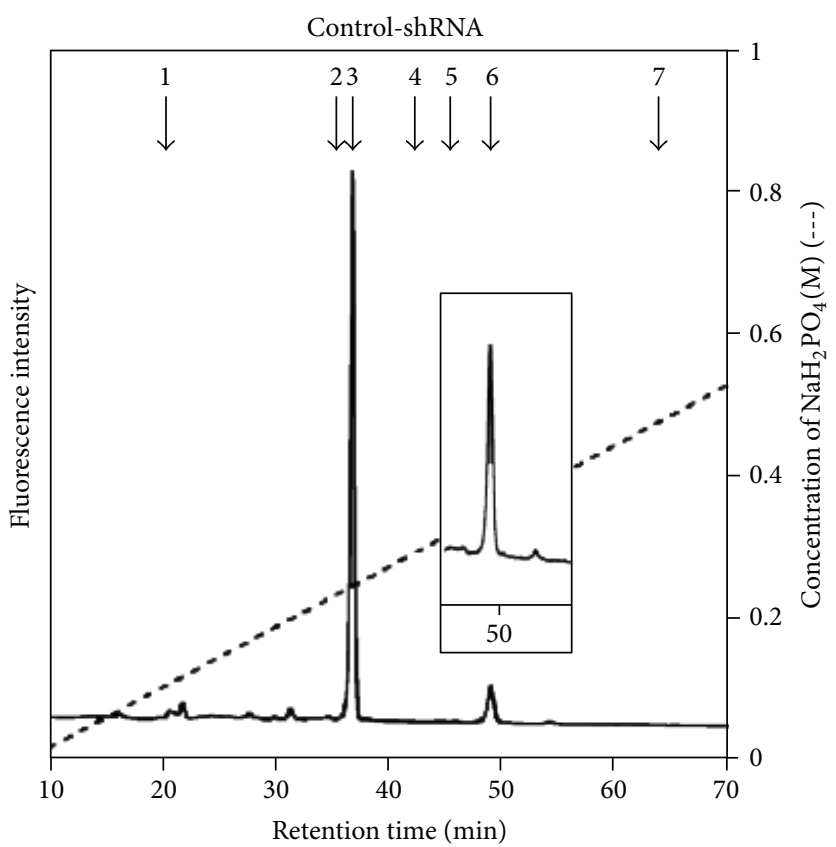

(b)

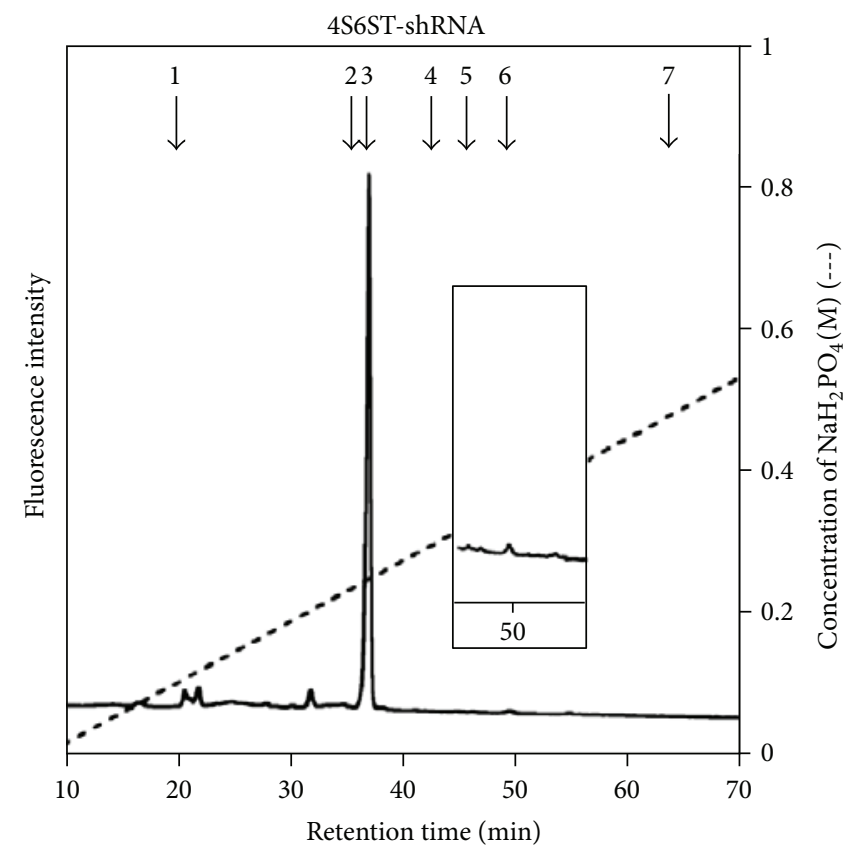

(c)

Figure 1: Quantitative real-time PCR analysis of the GalNAc4S-6ST transcript and profile of sulfation pattern of CS in the LLC-4S6STshRNA cells. (a) Total RNA was extracted from the control- (mock) or GalNAc4S-6ST-shRNA/LLC cells, and cDNA was synthesized by reverse transcriptase. Real-time PCR was conducted using the cDNAs, Taq polymerase, and SYBER Green. The expression of GalNAc4S-6ST was individually normalized to that of G3pdh. The assay was performed at least twice in triplicate, and representative results are shown. Values represent the mean $\pm \mathrm{SD} .{ }^{*} P<0.005 ;{ }^{* *} P<0.001$ versus control by one-way ANOVA with Dunnett's adjustment. (b, c) Anionexchange HPLC of disaccharides obtained from the digests of CS derived from control- and GalNAc4S-6ST-shRNA/LLC cells with a mixture of chondroitinases ABC and AC-II. The GAG-peptide preparations from LLC cells, which stably express control-shRNA (b) or 4S6STshRNA (c), were individually digested with a mixture of chondroitinases $\mathrm{ABC}$ and $\mathrm{AC}$-II. Each digest was labeled with a fluorophore $2 \mathrm{AB}$ as detailed in Section 2 and analyzed by anion-exchange HPLC on an amine-bound silica $\mathrm{PA}_{03}$ column using a linear gradient of $\mathrm{NaH}_{2} \mathrm{PO}_{4}$ as indicated by the dashed lines. The eluate was monitored by fluorescence intensity with the excitation and emission wavelengths of 330 and $420 \mathrm{~nm}$, respectively. The insets show magnified chromatograms (10-fold) around the elution position of $\triangle \mathrm{E}$ units. The positions of the $2 \mathrm{AB}-$ derivatized authentic disaccharides are indicated by numbered arrows: 1: $\triangle \mathrm{O}, \triangle \mathrm{HexUA-GalNAc;} 2: \triangle \mathrm{C}, \triangle \mathrm{HexUA-GalNAc}(6-\mathrm{O}$-sulfate); 3: $\Delta \mathrm{A}, \Delta \mathrm{HexUA}$-GalNAc(4-O-sulfate); 4: $\Delta \mathrm{D}, \Delta \mathrm{HexUA}(2-O$-sulfate)-GalNAc(6-O-sulfate); 5: $\Delta \mathrm{B}, \Delta \mathrm{HexUA}(2-O$-sulfate)-GalNAc(4-O-sulfate); 6: $\Delta \mathrm{E}, \Delta$ HexUA-GalNAc(4-O-, 6-O-disulfate); 7: $\Delta \mathrm{T}, \Delta \mathrm{HexUA}(2-O$-sulfate)-GalNAc(4-O-, 6-O-disulfate). 


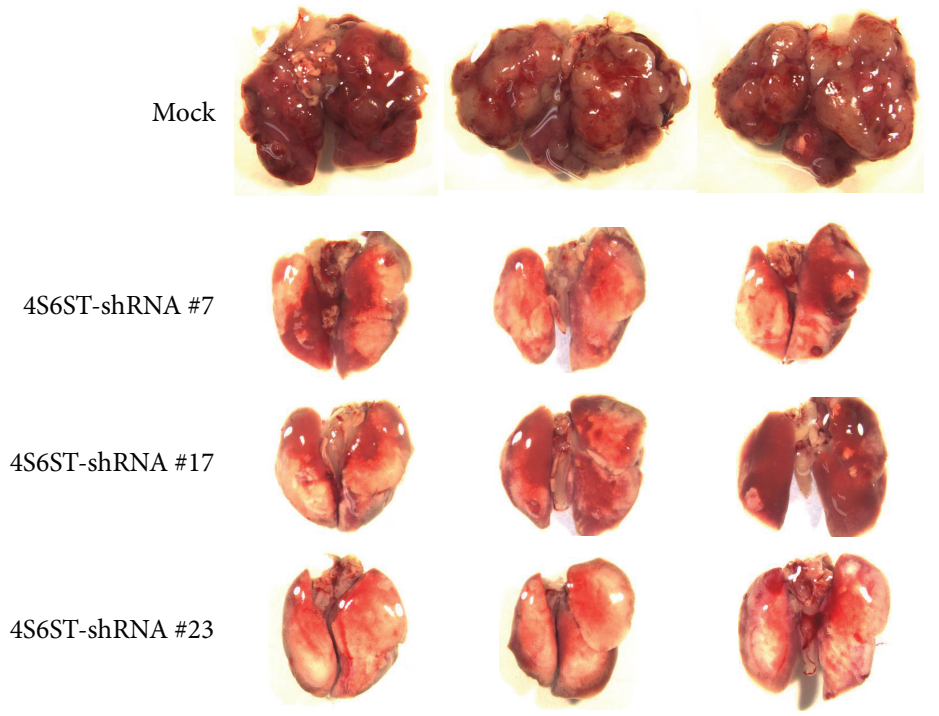

(a)

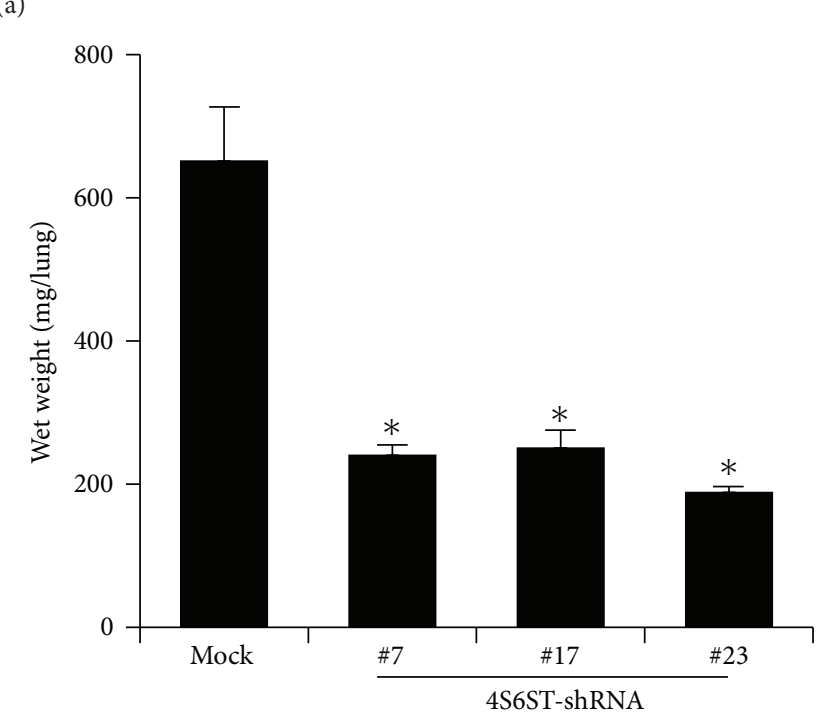

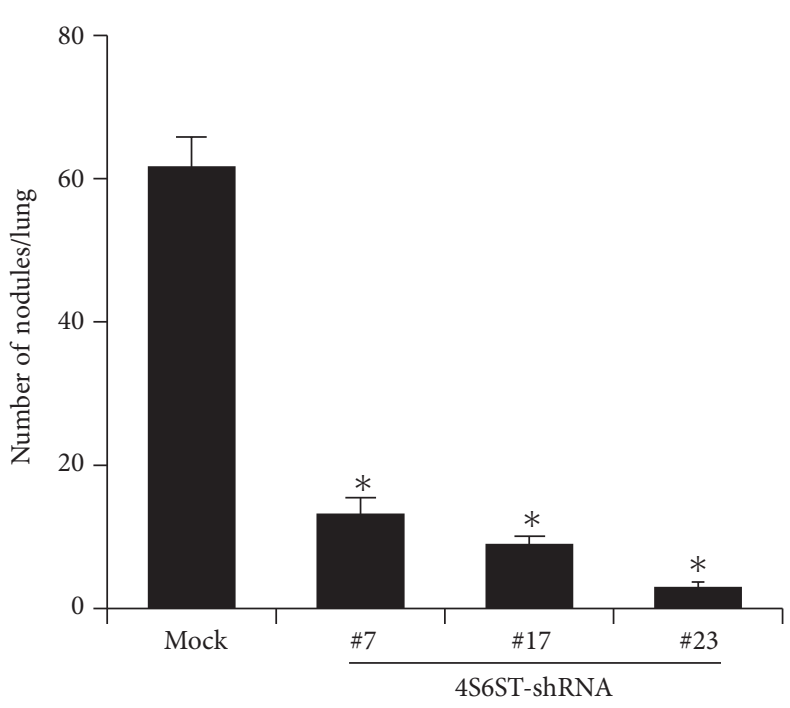

(b)

(c)

FIGURE 2: Effects of the knockdown of GalNAc4S-6ST on the pulmonary metastasis of LLC cells. Control- or GalNAc4S-6ST-shRNA/LLC-cell suspensions of $1 \times 10^{6}$ cells in $200 \mu \mathrm{L}$ of DMEM were injected into a tail vein of C57BL/6 J mice, and 21 days later, the number of tumor foci in the lungs was recorded. Six mice were used per group. Representative lungs from mice injected with the LLC cells stably expressing control(mock) or GalNAc4S-6ST-shRNA (4S6ST-shRNA) are shown in (a). The number of colonies (b) and the wet weight (c) of the lungs from mice injected with each LLC clone were measured. Data represent the mean \pm S.D. for two independent experiments. ${ }^{*} P<0.001$ versus control by one-way ANOVA with Dunnett's adjustment.

reduced (51 and $73 \%$ of the control-shRNA expressing LLC cells, resp.) (Figure 3). These observations indicate that CS chains at the LLC cell surface containing E-units may be involved in the initial cell adhesion to ECM molecules produced on the vascular endothelium in the lung during metastasis.

Along with the adhesion of LLC cells to a target tissue or cell, cell migration and invasion are also important to cancer progression and metastasis [35]. Next, to determine if the inhibition of the enzyme GalNAc4S-6ST using shRNA affects the migration and invasion of LLC cells; the invasive or migratory potential of LLC-4S6ST-shRNA cells was examined in vitro using a Boyden chamber coated with or without Matrigel, respectively. Although the migration was not significantly diminished by the knockdown of GalNAc4S6ST when compared with that using a control-shRNA (Figure 4(b)), down-regulation of E-units in CS chains caused by the knockdown of GalNAc4S-6ST resulted in a significant decrease in the invasion of LLC-4S6ST-shRNA cells through Matrigel as compared with the control knockdown (Figure 4(a)), indicating that GalNAc4ST-6ST and/or the E-units in CS chains regulate invasion but not migration during the metastasis of LLC cells, being consistent with a previous report [27]. 
TABLE 1: Disaccharide composition of CS chains in the control- and 4S6ST-shRNA/LLC cells. The GAG-peptide preparation from each cell line was digested with a mixture of chondroitinases $\mathrm{ABC}$ and $\mathrm{AC}-\mathrm{II}$ and analyzed by anion-exchange HPLC after labeling with a fluorophore $2 \mathrm{AB}$ as detailed in Section 2.

\begin{tabular}{|c|c|c|c|c|c|c|c|}
\hline & \multirow{2}{*}{ Wild type } & \multicolumn{3}{|c|}{ GalNAc4S-6ST-shRNA } & \multicolumn{3}{|c|}{ Control-shRNA } \\
\hline & & No. 7 & No. 17 & No. 23 & No. 5 & No. 10 & No. 14 \\
\hline & \multicolumn{7}{|c|}{$\mathrm{pmol} / \mathrm{mg}$ acetone powder $(\mathrm{mol} \%)^{\mathrm{b}}$} \\
\hline$\Delta \mathrm{A}^{\mathrm{a}}$ & $675(93.6)$ & $209(98.1)$ & $321(99.4)$ & $243(98.9)$ & $1,273(94.4)$ & $867(93.8)$ & $487(91.0)$ \\
\hline$\Delta \mathrm{E}^{\mathrm{a}}$ & $46(6.4)$ & $4(1.9)$ & $2(0.5)$ & $3(1.1)$ & $75(5.6)$ & $57(6.2)$ & $48(9.0)$ \\
\hline Total & $721(100)$ & $213(100)$ & $323(100)$ & $246(100)$ & $1,348(100)$ & $924(100)$ & $535(100)$ \\
\hline
\end{tabular}

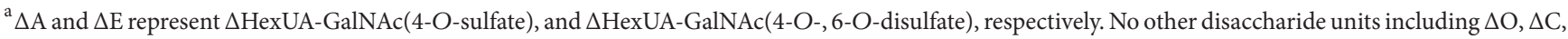
or $\Delta \mathrm{D}$ were detected (data not shown). $\Delta \mathrm{O}, \Delta \mathrm{C}$, and $\Delta \mathrm{D}$ stand for $\Delta \mathrm{HexUA-GalNAc,} \Delta \mathrm{HexUA-GalNAc(6-O-sulfate)}$ and $\Delta \mathrm{HexUA}(2-\mathrm{O}-\operatorname{sulfate}$-GalNAc(6O-disulfate), respectively.

${ }^{\mathrm{b}}$ Values are expressed in pmol of disaccharide per mg acetone powder as starting materials from the cells and calculated based on the peak areas of the disaccharides detected by anion-exchange HPLC (Figures 1(b) and 1(c)).

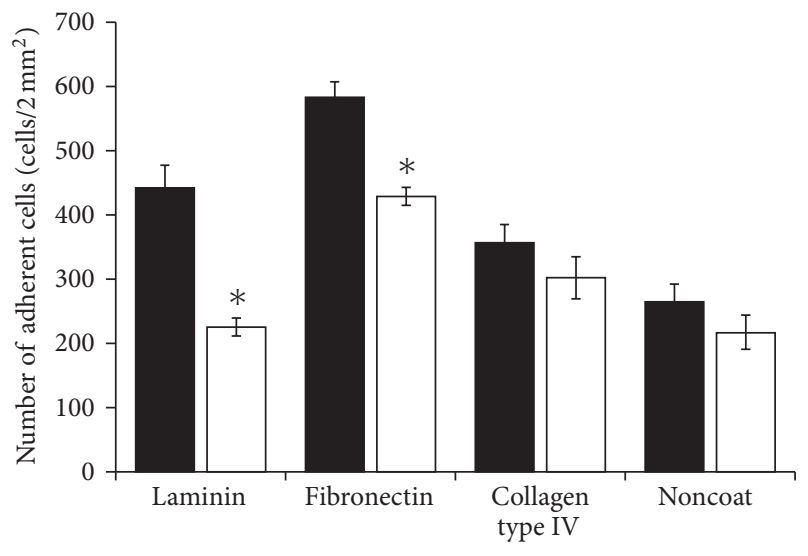

FIGURE 3: Effects of the knockdown of GalNAc4S-6ST on the adhesion of LLC cells. LLC cells stably expressing control-shRNA (filled bars) or GalNAc4S-6ST-shRNA (open bars) were seeded on laminin-, fibronectin-, or collagen type IV-precoated plastic cover slips for 1-2h. The cells were stained with Diff-Quik, and the adherent cells were counted. Average values obtained from three clones of both control-shRNA (nos. 5, 10, and 14) and GalNAc4S6ST-shRNA (nos. 7, 17, and 23) are shown, and the experiments were performed in triplicate. Error bars indicate \pm S.D. of triplicate samples. ${ }^{*} P<0.05$ versus control-shRNA by Student's $t$-test.

It is possible that LLC-4S6ST-shRNA cells are not so metastatic as we expect because of a possibility that they may grow more slowly than LLC-control-shRNA cells, which also partly contributes the lower metastatic capcity of LLC4S6ST-shRNA cells. To address this issue, the LLC cells were plated at a low density and the growth rate was determined. LLC-4S6ST-shRNA cells grew more slowly than control cells according to the results of a cell proliferation assay (Figure 5). These results indicate that the suppression of the metastasis of LLC cells by the knockdown of GalNAc4S-6ST shown in Figure 1(b) was partly due to a depression of cell growth potential.

\section{Discussion}

In the present study, to evaluate the involvement of E-units in CS chains expressed at the surface of LLC cells in tumor metastasis, we used an animal model of lung carcinoma and shRNA specific for the GalNAc4S-6ST gene, which is responsible for the formation of E-units [24, 25]. Firstly, GalNAc4S-6ST was overexpressed in LLC cells using several expression vectors such as pcDNA3.1/myc-His, pEF6/V5His, and pIRESneo3, although the expression of GalNAc4S6ST and E-units in CS chains was not enhanced for some unkown reason(s) (data not shown). Therefore, we assessed functions of E-units in the experimental metastatic model by knockdown of GalNAc4S-6ST using an shRNA-expressing vector in LLC cells.

CS-E interacts with heparin or heparan sulfatebinding proteins such as fibroblast growth factors, VEGF, pleiotrophin, and midkine [22, 36]. E-unit-containing structures of CS chains at the cell surface are important for the binding of LLC cells to laminin and fibronectin (Figure 3), which also interact with heparan sulfate [37-39]. Thus, E-units in CS chains in addition to heparan sulfate chains at the surface of LLC cells may also contribute the binding to laminin and/or fibronectin on the luminal side of the vascular endothelial cells in lungs.

Furthermore, endothelial carbohydrate-binding proteins, E- and P-selectins, play a role in the pulmonary metastasis of B16 melanoma cells [40]. It has also been reported that CS-E interacts with the adhesion molecules L- and P-selectins, and that CS-PGs at the surface of the metastatic breast cancer cell line are major P-selectin ligands on the endothelium [41, 42]. Hence, CS chains containing E-units may be involved in the adhesion of LLC cells through such cell adhesion molecules. In fact, most recently, we identified RAGE, which is a member of the immunoglobulin superfamily predominantly expressed in the lung, as a receptor for CS-E involved in pulmonary metastasis [28]. Together, the interaction of cell adhesion molecules or receptors including P-selectin and RAGE expressed on the endothelium at secondary target tissues with CS-containing E-units expressed on malignant cell surfaces play major or some roles in the targeting of tumor cells to lungs.

It has been demonstrated that matrix metalloproteinase9 is critical for the invasion and metastasis of LLC cells [43], and that heparan sulfate-proteoglycan, syndecan-2, functions as a suppressor for matrix metalloproteinase- 2 activation depending on the heparan sulfate side chains on LLC cells 


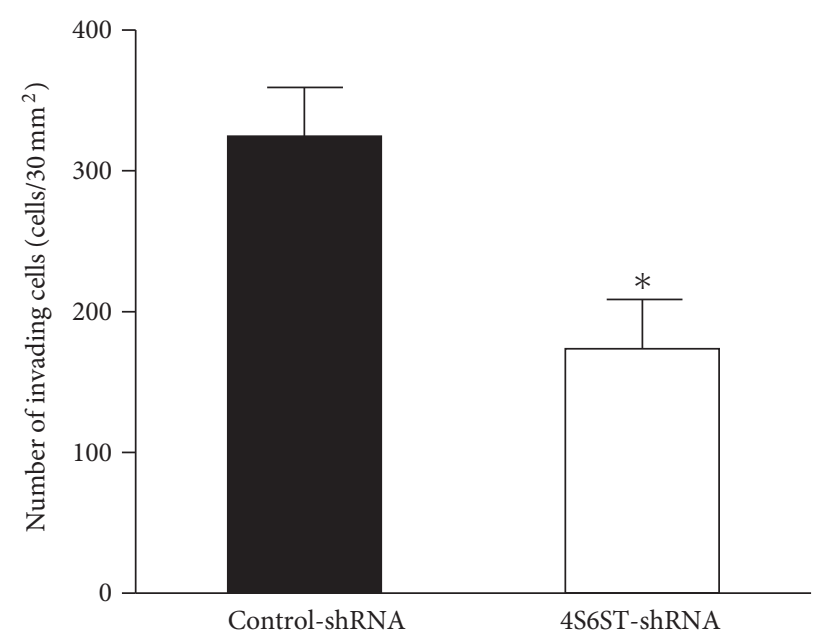

(a)

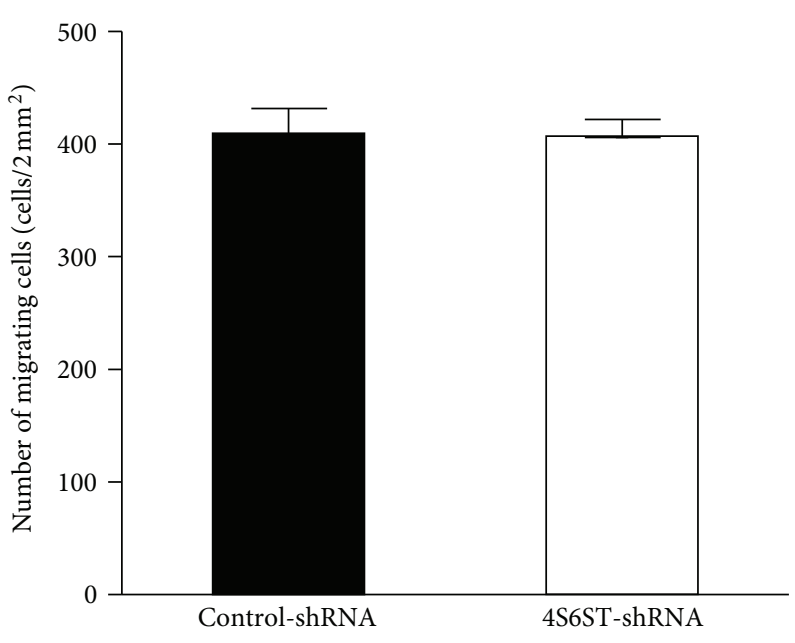

(b)

FIGURE 4: Effects of the knockdown of GalNAc4S-6ST on the invasion and migration of LLC cells. LLC cells stably expressing control-shRNA (filled bars) or GalNAc4S-6ST-shRNA (open bars) were plated on BD BioCoat chambers in the absence of fetal bovine serum. Cell invasion and migration were measured with or without Matrigel (BD Biosciences), respectively, as described in Section 2. Effects of the knockdown of GalNAc4ST-6ST on the invasion (a) and migration (b) of the LLC cells are summarized. The data represent the mean values \pm S.D. for three clones of both control-shRNA (nos. 5, 10, and 14) and GalNAc4S-6ST-shRNA (nos. 7, 17, and 23). Three independent experiments were performed, and the representative results are shown. ${ }^{*} P<0.05$ versus control-shRNA by Student's $t$-test.

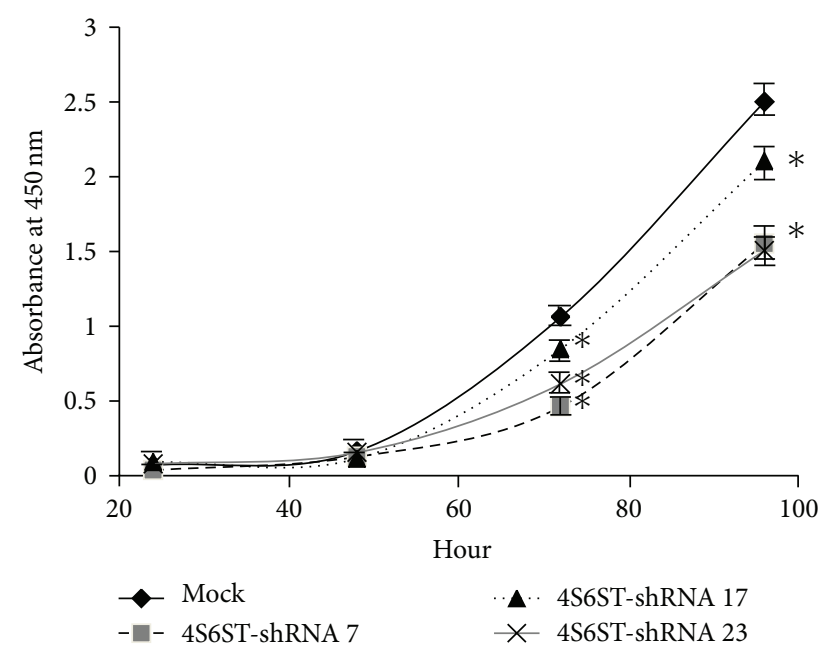

Figure 5: Effects of the knockdown of GalNAc4S-6ST on the proliferation of LLC cells. LLC cells stably expressing control-shRNA (mock) or GalNAc4S-6ST-shRNA (4S6ST-shRNA) were seeded on 96-well plates at $2 \times 10^{3}$ cells/well and incubated at $37^{\circ} \mathrm{C}$ for the assessed period. Cell numbers were measured every $24 \mathrm{~h}$ using TetraColor One reagent containing a tetrazolium and electronic carrier as described in Section 2. The data represent the mean \pm S.D. for three clones of both control-shRNA (nos. 5, 10, and 14) and GalNAc4S-6ST-shRNA (nos. 7, 17, and 23). Three independent experiments were performed, and representative results are shown. ${ }^{*} P<0.001$ versus control-shRNA by Student's $t$-test.

[44]. Moreover, melanoma CS-PG regulates membrane-type 3 matrix metalloproteinase and invasion of melanoma cells [45]. The invasive ability of the LLC-4S6ST-shRNA cells in Matrigel was lower than that of the LLC-control-shRNA cells
(Figure 4). Hence, CS-containing E-units as well as heparan sulfate may regulate metalloproteinases at the surfaces of LLC cells.

Metastasis is completed via processes involving growth, survival, and neoangiogenesis $[32,33]$. CS-E interacts with various heparin-binding proteins such as fibrobrast growth factors, midkine, and pleiotrophin [36]. Further, VEGF binds to CS-E expressed in tumor blood vessels in vitro [22]. Our findings (Figure 5) together with these observations prompted us to speculate that CS chains containing Eunits may participate as a regulator of VEGF signaling in the proliferation of tumor cells and tumor angiogenesis. In fact, GAG side chains including heparan sulfate and CS of neuropilin-1, a coreceptor for VEGF that augments angioplastic events through VEGF receptor-2, are critical for the reactivity to VEGF in endothelial cells and smooth muscle cells [46].

Interestingly, CS-PG, versican, functions as a macrophage activator that acts through Toll-like receptor-2, resulting in the production of tumor-necrosis factor- $\alpha$ and strong enhancement of the metastatic growth of LLC [10]. Notably, versican contains E-units in the CS side chains [42]. Thus, these observations with our results raise the possibility that E-unit-containing structures in the CS side chains of versican may contribute to the growth and/or metastasis of LLC cells.

\section{Conclusions}

In the present study, a reduction in the expression of GalNAc4S-6ST or the proportion of E-unit-containing CS chains effectively suppresses metastatic lung carcinoma through the reduction in adhesiveness, invasion, and proliferation but not the migration of LLC cells. Recently, we 
identified RAGE, which is specifically expressed in the normal lung, as a receptor for CS-E involved in the pulmonary metastasis [28]. The siRNA of the GalNAc4S-6ST gene and CS-E mimetics including small molecular inhibitors that bind RAGE are potential targets for anticancer therapies.

\section{Abbreviations}

$\begin{array}{ll}\text { 2AB: } & \text { 2-Aminobenzamide } \\ \text { CS: } & \text { Chondroitin sulfate } \\ \text { GAG: } & \text { Glycosaminoglycan } \\ \text { GalNAc: } & \text { N-Acetyl-D-galactosamine } \\ \text { GalNAc4S-6ST: } & \text { N-Acetylgalactosamine 4-O-sulfate } \\ & \text { 6-O-sulfotransferase } \\ \text { GlcUA: } & \text { D-Glucuronic acid } \\ \text { LLC: } & \text { Lewis lung carcinoma } \\ \text { PG: } & \text { Proteoglycan } \\ \text { shRNA: } & \text { Short hairpin RNA } \\ \text { VEGF: } & \text { Vascular endothelial growth factor. }\end{array}$

\section{Authors' Contribution}

S. Mizumoto and M. Watanabe contributed equally to this work.

\section{Acknowledgments}

This work was supported in part by Grant-in-Aid for Scientific Research (B) 23390016 (to K. Sugahara), Future Drug Discovery and Medical Care Innovation Program (to K. Sugahara), and Young Scientists (B) 23790066 (to S. Mizumoto) from Japan Society for the Promotion of Science (JSPS). None of these sponsors were involved in the experimental design.

\section{References}

[1] R. V. Iozzo, "Matrix proteoglycans: from molecular design to cellular function," Annual Review of Biochemistry, vol. 67, pp. 609-652, 1998.

[2] K. Sugahara and H. Kitagawa, "Recent advances in the study of the biosynthesis and functions of sulfated glycosaminoglycans," Current Opinion in Structural Biology, vol. 10, no. 5, pp. 518-527, 2000.

[3] K. Sugahara, T. Mikami, T. Uyama, S. Mizuguchi, K. Nomura, and H. Kitagawa, "Recent advances in the structural biology of chondroitin sulfate and dermatan sulfate," Current Opinion in Structural Biology, vol. 13, no. 5, pp. 612-620, 2003.

[4] K. Sugahara and T. Mikami, "Chondroitin/dermatan sulfate in the central nervous system," Current Opinion in Structural Biology, vol. 17, no. 5, pp. 536-545, 2007.

[5] M. M. Fuster and J. D. Esko, "The sweet and sour of cancer: glycans as novel therapeutic targets," Nature Reviews Cancer, vol. 5, no. 7, pp. 526-542, 2005.

[6] A. E. Faassen, J. A. Schrager, D. J. Klein, T. R. Oegema, J. R. Couchman, and J. B. McCarthy, "A cell surface chondroitin sulfate proteoglycan, immunologically related to $\mathrm{CD} 44$, is involved in type I collagen-mediated melanoma cell motility and invasion," Journal of Cell Biology, vol. 116, no. 2, pp. 521-531, 1992.
[7] U. Günthert, M. Hofmann, W. Rudy et al., "A new variant of glycoprotein CD44 confers metastatic potential to rat carcinoma cells," Cell, vol. 65, no. 1, pp. 13-24, 1991.

[8] J. Iida, A. M. L. Meijne, J. R. Knutson, L. T. Furcht, and J. B. McCarthy, "Cell surface chondroitin sulfate proteoglycans in tumor cell adhesion, motility and invasion," Seminars in Cancer Biology, vol. 7, no. 3, pp. 155-162, 1996.

[9] C. A. Cooney, F. Jousheghany, A. Yao-Borengasser et al., "Chondroitin sulfates play a major role in breast cancer metastasis: a role for CSPG4 and CHST11 gene expression in forming surface P-selectin ligands in aggressive breast cancer cells," Breast Cancer Research, vol. 13, article R58, 2011.

[10] S. Kim, H. Takahashi, W. W. Lin et al., "Carcinoma-produced factors activate myeloid cells through TLR2 to stimulate metastasis," Nature, vol. 457, no. 7225, pp. 102-106, 2009.

[11] M. Kusche-Gullberg and L. Kjellén, "Sulfotransferases in glycosaminoglycan biosynthesis," Current Opinion in Structural Biology, vol. 13, no. 5, pp. 605-611, 2003.

[12] M. Klüppel, T. N. Wight, C. Chan, A. Hinek, and J. L. Wrana, "Maintenance of chondroitin sulfation balance by chondroitin4 -sulfotransferase 1 is required for chondrocyte development and growth factor signaling during cartilage morphogenesis," Development, vol. 132, no. 17, pp. 3989-4003, 2005.

[13] H. Thiele, M. Sakano, H. Kitagawa et al., "Loss of chondroitin 6-O-sulfotransferase-1 function results in severe human chondrodysplasia with progressive spinal involvement," Proceedings of the National Academy of Sciences of the United States of America, vol. 101, no. 27, pp. 10155-10160, 2004.

[14] M. H. H. van Roij, S. Mizumoto, S. Yamada et al., "Spondyloepiphyseal dysplasia, omani type: further definition of the phenotype," American Journal of Medical Genetics A, vol. 146, no. 18, pp. 2376-2384, 2008.

[15] B. Tuysuz, S. Mizumoto, K. Sugahara, A. Çelebi, S. Mundlos, and S. Turkmen, "Omani-type spondyloepiphyseal dysplasia with cardiac involvement caused by a missense mutation in CHST3," Clinical Genetics, vol. 75, no. 4, pp. 375-383, 2009.

[16] S. Nadanaka, A. Clement, K. Masayama, A. Faissner, and K. Sugahara, "Characteristic hexasaccharide sequences in octasaccharides derived from shark cartilage chondroitin sulfate D with a neurite outgrowth promoting activity," Journal of Biological Chemistry, vol. 273, no. 6, pp. 3296-3307, 1998.

[17] X. Bao, T. Muramatsu, and K. Sugahara, "Demonstration of the pleiotrophin-binding oligosaccharide sequences isolated from chondroitin sulfate/dermatan sulfate hybrid chains of embryonic pig brains," Journal of Biological Chemistry, vol. 280, no. 42, pp. 35318-35328, 2005.

[18] A. Purushothaman, K. Sugahara, and A. Faissner, "Chondroitin sulfate, "wobble motifs" modulate maintenance and differentiation of neural stem cells and their progeny," Journal of Biological Chemistry, vol. 287, no. 5, pp. 2935-2942, 2012.

[19] K. Bergefall, E. Trybala, M. Johansson et al., "Chondroitin sulfate characterized by the E-disaccharide unit is a potent inhibitor of herpes simplex virus infectivity and provides the virus binding sites on gro2C cells," Journal of Biological Chemistry, vol. 280, no. 37, pp. 32193-32199, 2005.

[20] T. Uyama, M. Ishida, T. Izumikawa et al., "Chondroitin 4$O$-sulfotransferase-1 regulates $\mathrm{E}$ disaccharide expression of chondroitin sulfate required for herpes simplex virus infectivity," Journal of Biological Chemistry, vol. 281, no. 50, pp. 38668-38674, 2006. 
[21] A. Purushothaman, J. Fukuda, S. Mizumoto et al., "Functions of chondroitin sulfate/dermatan sulfate chains in brain development: critical roles of $\mathrm{E}$ and $\mathrm{iE}$ disaccharide units recognized by a single chain antibody GD3G7," Journal of Biological Chemistry, vol. 282, no. 27, pp. 19442-19452, 2007.

[22] G. B. ten Dam, E. M. A. van de Westerlo, A. Purushothaman et al., "Antibody GD3G7 selected against embryonic glycosaminoglycans defines chondroitin sulfate-E domains highly up-regulated in ovarian cancer and involved in vascular endothelial growth factor binding," American Journal of Pathology, vol. 171, no. 4, pp. 1324-1333, 2007.

[23] K. N. Sugahara, T. Hirata, T. Tanaka et al., "Chondroitin sulfate E fragments enhance CD44 cleavage and CD44-dependent motility in tumor cells," Cancer Research, vol. 68, no. 17, pp. 7191-7199, 2008.

[24] S. Ohtake, Y. Ito, M. Fukuta, and O. Habuchi, "Human $\mathrm{N}$-acetylgalactosamine 4-sulfate 6-O-sulfotransferase cDNA is related to human $\mathrm{B}$ cell recombination activating geneassociated gene," Journal of Biological Chemistry, vol. 276, no. 47, pp. 43894-43900, 2001.

[25] S. Ohtake-Niimi, S. Kondo, T. Ito et al., "Mice deficient in $\mathrm{N}$-acetylgalactosamine 4-sulfate 6-O-sulfotransferase are unable to synthesize chondroitin/dermatan sulfate containing $\mathrm{N}$-acetylgalactosamine 4,6-bissulfate residues and exhibit decreased protease activity in bone marrow-derived mast cells," Journal of Biological Chemistry, vol. 285, no. 27, pp. 20793-20805, 2010.

[26] Y. Ito, M. Watanabe, T. Nishizawa et al., "The utility of formalinfixed and paraffin-embedded tissue blocks for quantitative analysis of $\mathrm{N}$-acetylgalactosamine 4 -sulfate 6-O-sulfotransferase mRNA expressed by colorectal cancer cells," Acta Histochemica et Cytochemica, vol. 40, no. 2, pp. 53-59, 2007.

[27] F. Li, G. B. ten Dam, S. Murugan et al., "Involvement of highly sulfated chondroitin sulfate in the metastasis of the Lewis lung carcinoma cells," Journal of Biological Chemistry, vol. 283, no. 49, pp. 34294-34304, 2008.

[28] S. Mizumoto, J. Takahashi, and K. Sugahara, "Receptor for advanced glycation end products (RAGE) functions as receptor for specific sulfated glycosaminoglycans, and anti-RAGE antibody or sulfated glycosaminoglycans delivered in vivo inhibit pulmonary metastasis of tumor cells," Journal of Biological Chemistry, vol. 287, no. 23, pp. 18985-18994, 2012.

[29] A. Kinoshita and K. Sugahara, "Microanalysis of glycosaminoglycan-derived oligosaccharides labeled with a fluorophore 2-aminobenzamide by high-performance liquid chromatography: application to disaccharide composition analysis and exosequencing of oligosaccharides," Analytical Biochemistry, vol. 269, no. 2, pp. 367-378, 1999.

[30] S. Mizumoto and K. Sugahara, "Glycosaminoglycan chain analysis and characterization (glycosylation/epimerization)," Methods in Molecular Biology, vol. 836, pp. 99-115, 2012.

[31] B. Rashidi, M. Yang, P. Jiang et al., "A highly metastatic Lewis lung carcinoma orthotopic green fluorescent protein model," Clinical and Experimental Metastasis, vol. 18, no. 1, pp. 57-60, 2000.

[32] I. J. Fidler, “The pathogenesis of cancer metastasis: the 'seed and soil' hypothesis revisited," Nature Reviews Cancer, vol. 3, no. 6, pp. 453-458, 2003.

[33] P. S. Steeg, "Tumor metastasis: mechanistic insights and clinical challenges," Nature Medicine, vol. 12, no. 8, pp. 895-904, 2006.
[34] S. A. Eccles and D. R. Welch, "Metastasis: recent discoveries and novel treatment strategies," The Lancet, vol. 369, no. 9574, pp. 1742-1757, 2007.

[35] P. Friedl and K. Wolf, "Tumour-cell invasion and migration: diversity and escape mechanisms," Nature Reviews Cancer, vol. 3, no. 5, pp. 362-374, 2003.

[36] S. S. Deepa, Y. Umehara, S. Higashiyama, N. Itoh, and K. Sugahara, "Specific molecular interactions of oversulfated chondroitin sulfate $\mathrm{E}$ with various heparin-binding growth factors: implications as a physiological binding partner in the brain and other tissues," Journal of Biological Chemistry, vol. 277, no. 46, pp. 43707-43716, 2002.

[37] E. Ruoslahti and E. Engvall, "Complexing of fibronectin glycosaminoglycans and collagen," Biochimica et Biophysica Acta, vol. 631, no. 2, pp. 350-358, 1980.

[38] E. G. Hayman, A. Oldberg, G. R. Martin, and E. Ruoslahti, "Codistribution of heparan sulfate proteoglycan, laminin, and fibronectin in the extracellular matrix of normal rat kidney cells and their coordinate absence in transformed cells," Journal of Cell Biology, vol. 94, no. 1, pp. 28-35, 1982.

[39] M. P. Hoffman, J. A. Engbring, P. K. Nielsen et al., "Cell type-specific differences in glycosaminoglycans modulate the biological activity of a heparin-binding peptide (RKRLQVQLSIRT) from the G domain of the laminin $\alpha 1$ chain," Journal of Biological Chemistry, vol. 276, no. 25, pp. 22077-22085, 2001.

[40] J. Zhang, J. Nakayama, C. Ohyama et al., "Sialyl Lewis Xdependent lung colonization of B16 melanoma cells through a selectin-like endothelial receptor distinct from E- or P-selectin," Cancer Research, vol. 62, no. 15, pp. 4194-4198, 2002.

[41] B. Monzavi-Karbassi, J. S. Stanley, L. Hennings et al., "Chondroitin sulfate glycosaminoglycans as major P-selectin ligands on metastatic breast cancer cell lines," International Journal of Cancer, vol. 120, no. 6, pp. 1179-1191, 2007.

[42] H. Kawashima, K. Atarashi, M. Hirose et al., "Oversulfated chondroitin/dermatan sulfates containing GlcA $\beta 1 / \operatorname{IdoA} \alpha 1$ $3 \mathrm{GalNAc}(4,6-\mathrm{O}$-disulfate) interact with $\mathrm{L}$ - and P-selectin and chemokines," Journal of Biological Chemistry, vol. 277, no. 15, pp. 12921-12930, 2002.

[43] C. H. Chou, C. M. Teng, K. Y. Tzen, Y. C. Chang, J. H. Chen, and J. C. H. Cheng, "MMP-9 from sublethally irradiated tumor promotes Lewis lung carcinoma cell invasiveness and pulmonary metastasis," Oncogene, vol. 31, pp. 458-468, 2011.

[44] S. Munesue, Y. Yoshitomi, Y. Kusano et al., "A novel function of syndecan-2, suppression of matrix metalloproteinase-2 activation, which causes suppression of metastasis," Journal of Biological Chemistry, vol. 282, no. 38, pp. 28164-28174, 2007.

[45] J. Iida, D. Pei, T. Kang et al., "Melanoma chondroitin sulfate proteoglycan regulates matrix metalloproteinase-dependent human melanoma invasion into type I collagen," Journal of Biological Chemistry, vol. 276, no. 22, pp. 18786-18794, 2001.

[46] Y. Shintani, S. Takashima, Y. Asano et al., "Glycosaminoglycan modification of neuropilin-1 modulates VEGFR2 signaling," EMBO Journal, vol. 25, no. 13, pp. 3045-3055, 2006. 

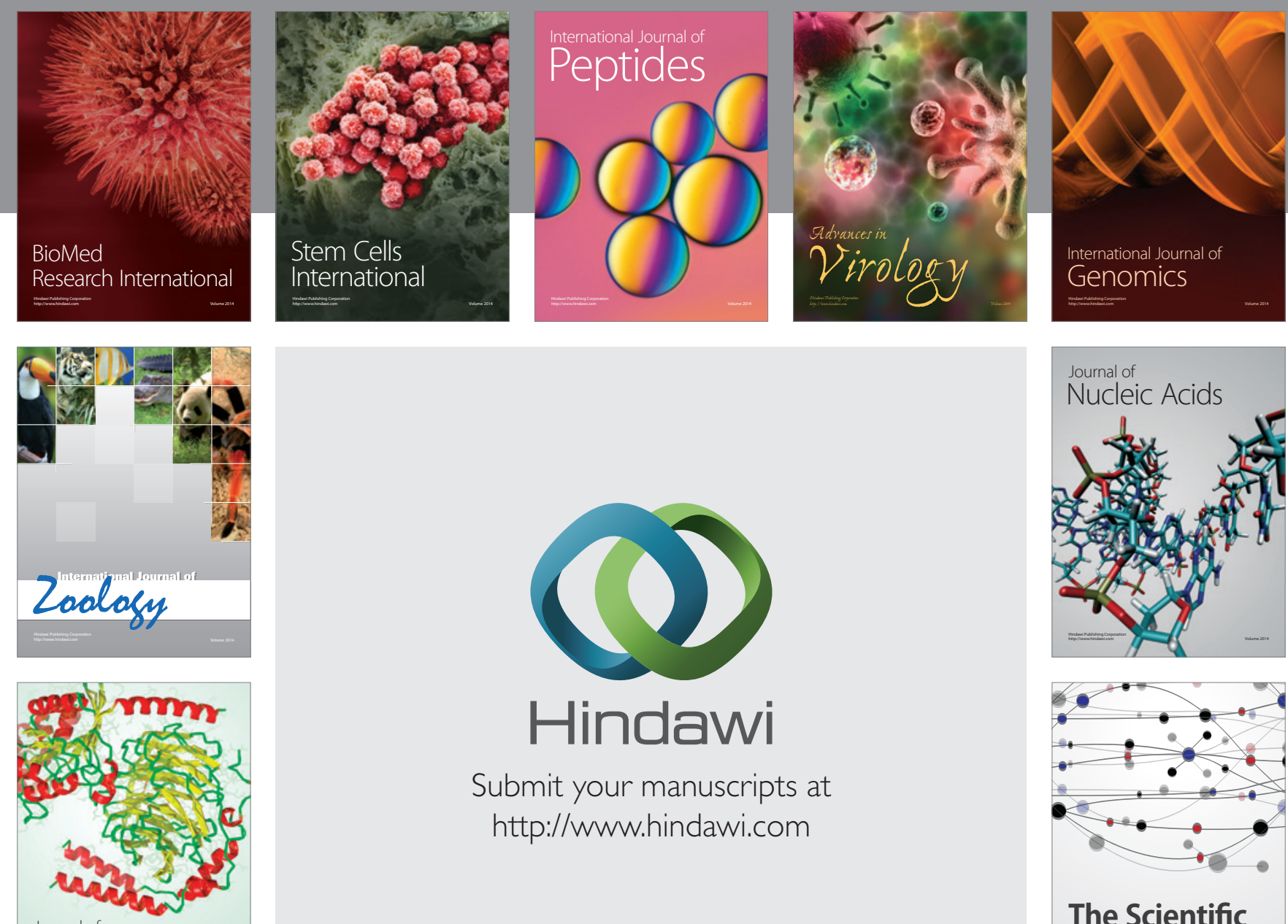

Submit your manuscripts at

http://www.hindawi.com

Journal of
Signal Transduction
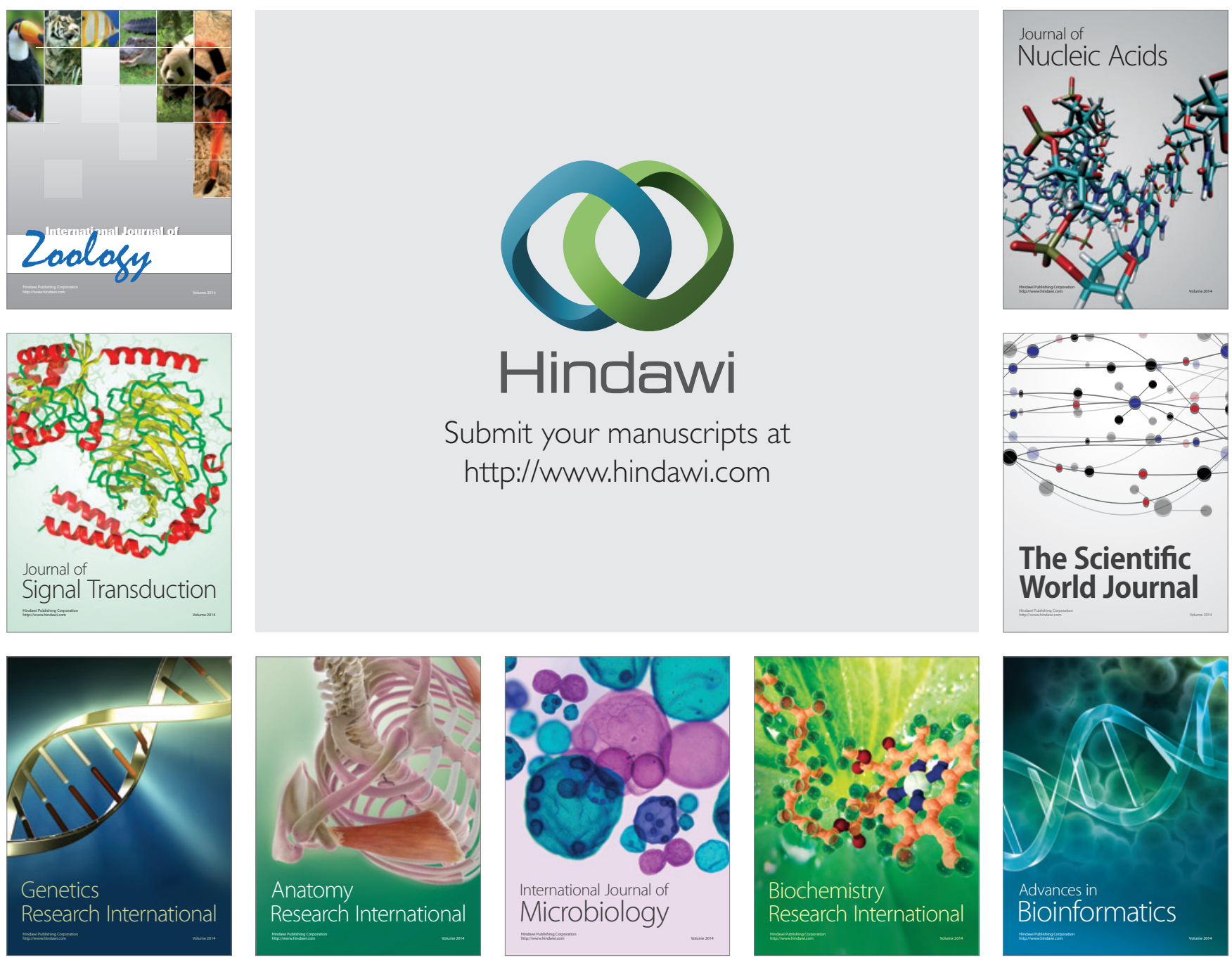

The Scientific World Journal
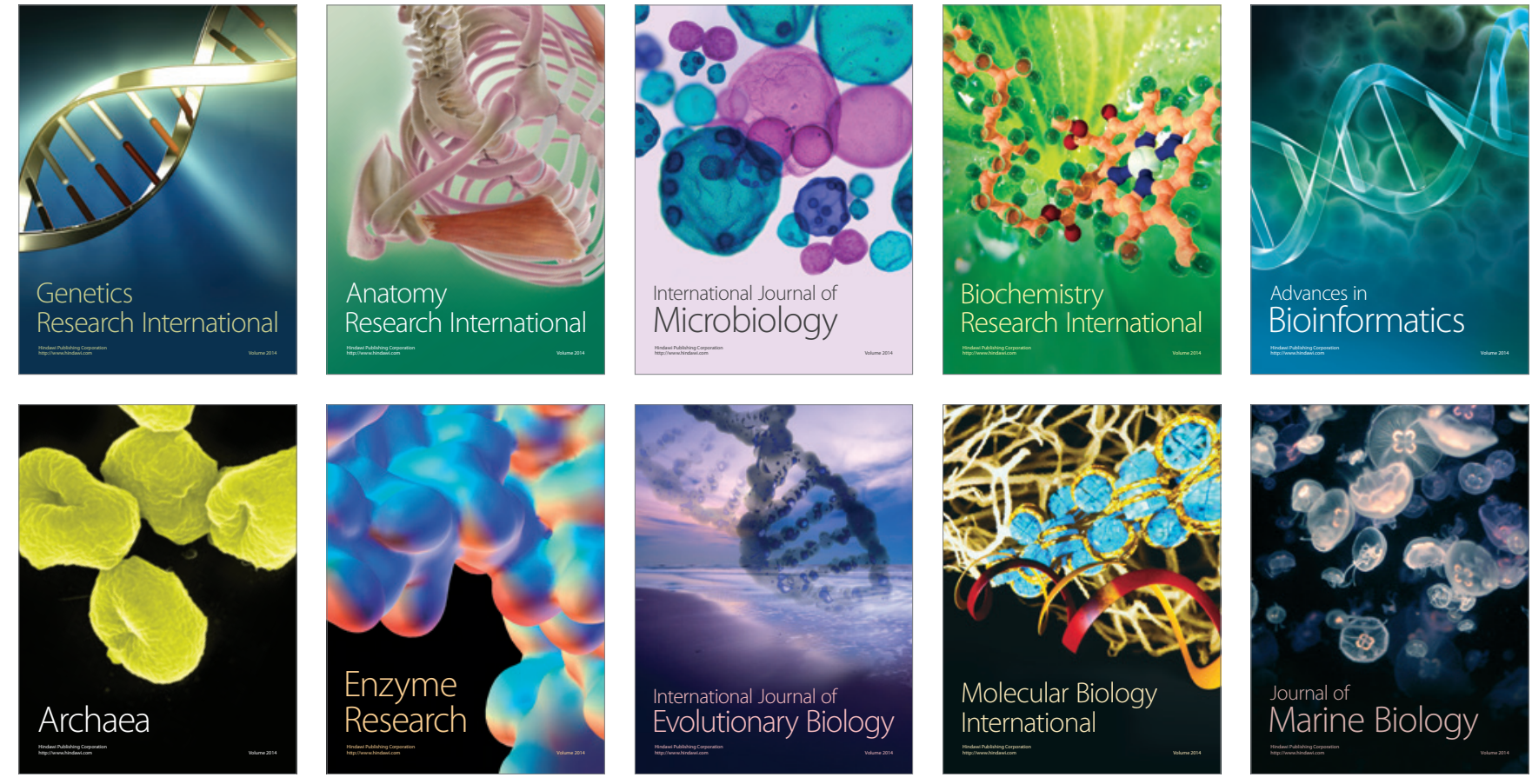